\title{
Intrusion history of the Portrush Sill, County Antrim, Northern Ireland: evidence for rapid emplacement and high-temperature contact metamorphism
}

\author{
MORGANE LEDEVIN* ${ }^{*}$, NICHOLAS ARNDT*, MARK R. COOPER \\ GARTH EARLS $\ddagger$, PAUL LYLE $\ddagger$, CHARLES AUBOURG $\S \&$ ERIC LEWIN* \\ * Institut des Sciences de la Terre (ISTerre), Université de Grenoble, Grenoble 38401, France \\ $\ddagger$ Geological Survey of Northern Ireland, Colby House, Stranmillis Court, Belfast, Northern Ireland, BT9 5BF, UK \\ $\S$ Université Cergy-Pontoise, 5 mail Gay-Lussac Neuville-sur-Oise, F-95031 Cergy-Pontoise
}

(Received 28 July 2010; accepted 15 February 2011; first published online 19 September 2011)

\begin{abstract}
The gabbroic Portrush Sill in Northern Ireland, part of the North Atlantic Igneous Province, intruded Lower Jurassic mudstones and siltstones about $55 \mathrm{Ma}$ ago. We used petrologic observations and geochemical analyses to study how the sill interacted with the sedimentary rocks. Field relationships show that an Upper Sill and numerous associated Minor Intrusions were emplaced in the sedimentary host rocks before intrusion of the Main Sill, some $10 \mathrm{~m}$ above its upper contact. Geochemical analyses reveal two magma contamination processes: $\mathrm{Nb}$ and $\mathrm{Ta}$ anomalies, coupled with incompatible element enrichment, record contamination by deep crustal rocks, whereas $\mathrm{Li}, \mathrm{Pb}$ and $\mathrm{Ba}$ anomalies reveal a superficial contamination through fluid circulation at the contact between magmatic and sedimentary rocks. Analysis of mineral assemblages and geochemical data from the contact aureole demonstrate uniform metamorphic conditions between the two main intrusions and an absence of a thermal gradient. The identification of pyrrhotite by magnetization analyses and of orthopyroxene by microprobe analyses indicates very high temperatures, up to $660^{\circ} \mathrm{C}$. Thermal modelling explains these temperatures as the coupled effects of the Main Sill and the earlier intruded Upper Sill and Minor Intrusions. Even though the chemical composition of the Main Sill suggests another type of parental liquid, all three units were emplaced in a very short time, certainly less than five years.
\end{abstract}

Keywords: Portrush Sill, contact metamorphism, magma contamination, thermal modelling, Northern Ireland.

\section{Introduction}

The North Atlantic Igneous Province was emplaced over several million years, during the peak of activity of the precursor of the Icelandic plume. The initial stages preceded the opening of North Atlantic Ocean $55 \mathrm{Ma}$ ago and were accompanied by the eruption of flood basalts on both sides of the ocean basin (McHone \& Butler, 1984; White, 1989; Coffin \& Eldholm, 1994; White \& McKenzie, 1995; Courtillot et al. 1999; Scarrow, Curran \& Kerr, 2000; Pirajno, 2004; Sigmundsson, 2006; Coulon, 2003; Landes, Ritter \& Readman, 2007). The segment of flood basalts preserved in Northern Ireland, known as the Antrim Lava Group, has been recently dated using the ${ }^{40} \mathrm{Ar}-$ ${ }^{39} \mathrm{Ar}$ method at $61.4 \pm 0.4 \mathrm{Ma}$ for the lower units and $59 \pm 0.4 \mathrm{Ma}$ for the upper unit (McKenna, 2009; Ganerød et al. 2008). The eruption of these basalts is close to the Paleocene-Eocene boundary, a period of pronounced warming and a marked change in carbon isotopic composition (Kennett \& Scott, 1991; Thomas \& Shackelton, 1996; Norris \& Röhl, 1999; Rhöl et al. 2000; Farley \& Eltgroth, 2003; Schmitz et al. 2004; Tripati \& Elderfield, 2005; Zachos et al. 2005; Storey,

$\dagger$ Author for correspondence: morgane.ledevin@ujf-grenoble.fr
Duncan \& Swisher, 2007). Svensen et al. (2004, 2007) attributed such changes to the expulsion of toxic or greenhouse gases from the contact aureoles surrounding intrusions of the igneous province.

The Antrim Lava Group and underlying sediment pile are cut by numerous shallow mafic intrusions, one of which is the Portrush Sill. This intrusion became famous in the 18 th century because of a debate surrounding the interpretation of ammonite-bearing hornfels at its upper contact. Richardson (1803), a follower of Werner, the Neptunist, maintained that these hard, fine-grained rocks were basalts and used the presence of the fossils within them to argue for a sedimentary origin. Playfair (1802), who subscribed to the ideas of Hutton the Plutonist, recognized that they formed part of the contact aureole that surrounded the sill and used the evidence of intense heating of the sedimentary rocks to argue for a high-temperature origin as a magmatic rock.

In this study, we sampled in detail the contact aureole in order to determine how the Portrush Sill interacted with its sedimentary host rocks. Our field and petrographic studies revealed the complex structure of this intrusion and the presence of two new magmatic units. We thus provide an example of comagmatic intrusions emplaced in a very short time, 


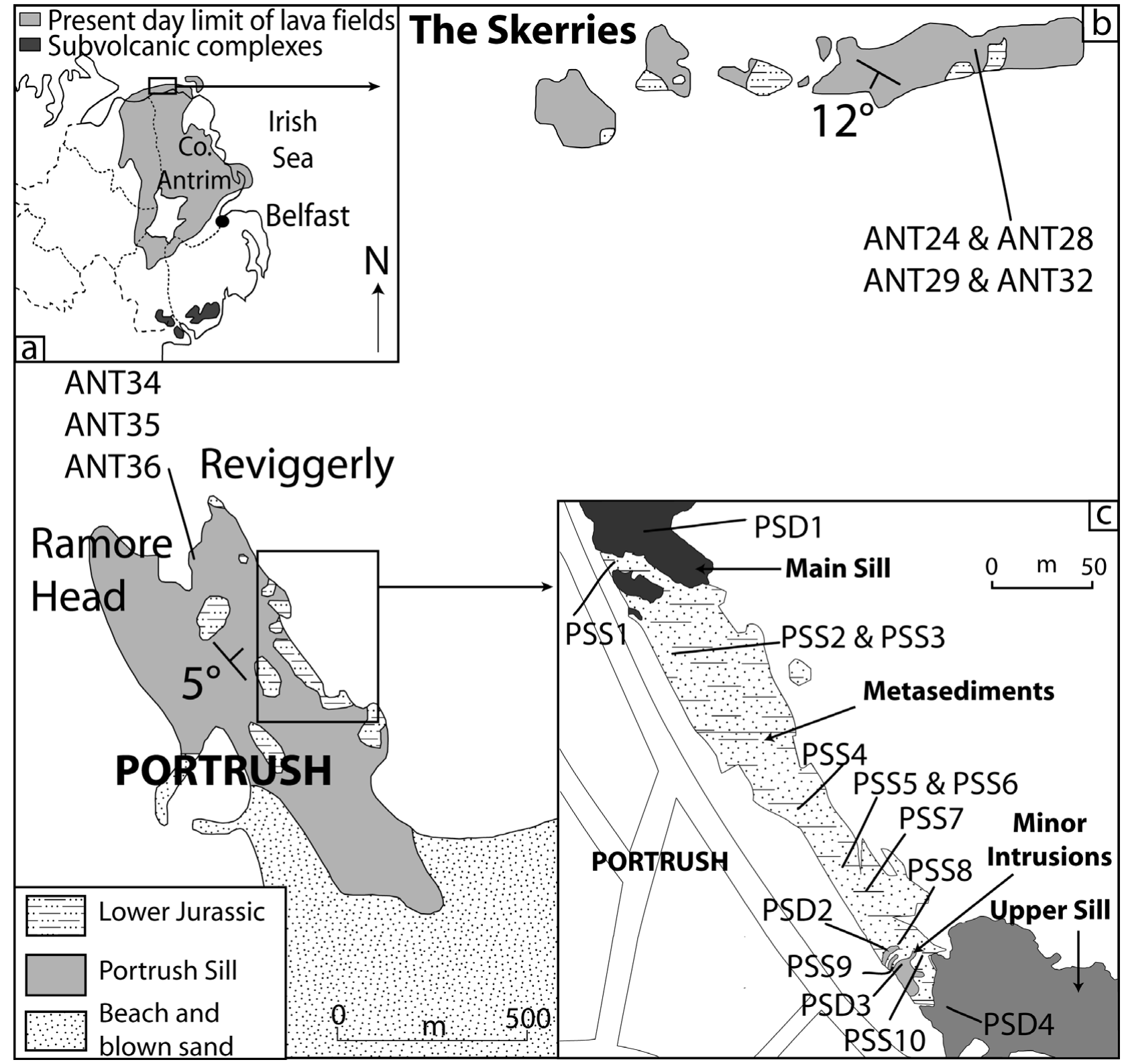

Figure 1. (a) Simplified map of Northern Ireland, modified from Mitchell (2004), showing the study area. (b) Geological map of the Portrush Sill area, modified from Hawkes \& Wilson (1975). (c) Detail of the Portrush waterfront: geological map showing Main Sill, Upper Sill, Minor Intrusions and metasediments. Sample sites are shown in (b) and (c).

with the consequence that very high temperatures were produced in the sedimentary rocks between the two main units.

\section{Geological background}

In Late Cretaceous to Early Paleocene time, the North Atlantic region saw the arrival of a mantle plume at the base of the continental lithosphere. Partial melting in the plume resulted in the production of large volumes of magma that formed the North Atlantic Igneous Province, which extended to the west of Greenland, offshore Norway, NW Scotland and Northern Ireland (Coffin \& Eldholm, 1994; White \& McKenzie, 1995; Scarrow, Curran \& Kerr, 2000; Pirajno, 2004; Landes, Ritter \& Readman, 2007). Around $65 \mathrm{Ma}$, the whole region underwent rifting that resulted in the divergence of the North American and Eurasian plates and the opening of the North Atlantic at $55 \mathrm{Ma}$ (Hallam, 1971; McHone \& Butler, 1984; White, 1989; Courtillot et al. 1999; Coulon, 2003; Sigmundsson, 2006). During these events, the Icelandic plume exhibited an irregular activity with one pulse at $62 \mathrm{Ma}$ and two others at 59 and $55 \mathrm{Ma}$. Forming the Antrim Plateau in Northern Ireland, the Antrim Lava Group erupted during these peaks, together with contemporaneous intrusive complexes, dykes and sills (Patterson, 1955; Old, 1975; McHone \& Butler, 1984; Thompson, 1985; Lyle, 2003; Mitchell, 2004).

The Portrush Sill is located on the north coast of County Antrim (Fig. 1a) and forms the Ramore Head Peninsula in the town of Portrush. It extends to the north for $2 \mathrm{~km}$ offshore to form the E-W alignment of islands known as the Skerries (Fig. 1b). The sill is 
apparently lopolithic (Hawkes \& Wilson, 1975) and its thickness remains unknown: the base of the intrusion is not exposed but its thickness is estimated at between $45 \mathrm{~m}$ (Mitchell, 2004) and $>60 \mathrm{~m}$ (Hawkes \& Wilson, 1975). McKenna (2009) dated the intrusion using the ${ }^{40} \mathrm{Ar}-{ }^{39} \mathrm{Ar}$ method and obtained an age of $54.9 \pm$ 0.6 Ma, making the intrusion slightly younger than most of the volcanic rocks.

Hawkes \& Wilson (1975) described the petrology and mineralogy of the lithological units within the sill and the hornfelsed Jurassic sedimentary rocks at its upper contact. In the sill they recognized three main divisions of dolerite or gabbro that can be distinguished by their structure and mineralogy with a mean composition of 0 to $12 \%$ olivine, 50 to $60 \%$ plagioclase, 20 to $30 \%$ pyroxene and 2 to $6 \%$ oxides. From their field observations, they reported that the Jurassic sediments are baked to nearly $8 \mathrm{~m}$ beyond the contact with the sill, but they did not describe the overlying magmatic units we will present in this paper.

\section{Sampling and analytical methods}

To analyse both the igneous rocks of the Portrush Sill and the hornfelsed sedimentary rocks of the contact aureole, we sampled along a profile crossing these units (Fig. 1b, c; see Fig. 4 for GPS locations). Before undertaking this sampling, a detailed study was made in the field of the units overlying the Main Sill. Igneous samples were collected across the Main Sill (ANT34 to 36), from smaller dolerite units that intruded the overlying metasediments (PSD1 to 3), and from another magmatic unit that overlies the whole sequence (PSD4) (Fig. 1b, c). A total of ten sedimentary samples were collected from the $10 \mathrm{~m}$ of baked Lower Jurassic rocks above the Main Sill (PSS1 to 10). Finally, a small dolerite intrusion on the Large Skerries was sampled along a profile cutting through the intrusion (ANT24 \& ANT28) and the surrounding hornfelsed host rocks (ANT29 \& ANT32), with the aim of comparing the results with the Portrush profile.

All the samples were studied in thin-section and by X-ray diffraction spectrometry, augmented by microprobe analyses, to determine the major mineral phases in the fine-grained hornfels. All analyses were performed in the geochemistry department (ISTerre) of the University of Grenoble, France. Measurements of remnant magnetization were conducted at the Parc Saint-Maur Observatory in Paris and used to investigate the nature of magnetic minerals in the metasedimentary rocks.

Major and trace elements were analysed at ISTerre. Sample preparation and measurements of loss-onignition (LOI) were performed in a clean room and the assays were performed by inductively coupled plasma atomic emission spectrometry (ICP-AES) and inductively coupled plasma mass spectrometry (ICPMS) using techniques described by Chauvel, Bureau $\&$ Poggi (2011). The uncertainty on the major element data is estimated at $5 \%$ and given as a percentage of standard weight of oxide. Errors on trace-element data are estimated at $5 \%$. Note that some concentrations, such as phosphorus $(\mathrm{P})$ and cadmium $(\mathrm{Cd})$, were below the detection limit of the machine, and could not be analysed.

\section{Field relationships}

In outcrops on Ramore Head, Reviggerly and the waterfront of Portrush (Fig. 1b), several gabbroic to doleritic units intrude the metasedimentary rocks that immediately overlie the Main Sill, as shown in Figures 1, 2 and 3. The uppermost and thickest of these minor intrusions, an olivine dolerite that we will call the Upper Sill, intruded about $10 \mathrm{~m}$ above the Main Sill (Fig. 3). The upper part of this unit has been removed by erosion and its full thickness is unknown; however, the rock is medium grained throughout and it was probably not much thicker than the $15 \mathrm{~m}$ that remains. Other smaller doleritic intrusions, which we refer to as the Minor Intrusions, form a complex of irregular units intruding the metasediments between the Upper Sill and the Main Sill. They first appear about $6 \mathrm{~m}$ above the upper contact of the Main Sill (Fig. 2d, e) and persist upward to intrude the Upper Sill (Fig. 2b, c). Their thickness varies from tens of centimetres to a metre. They are broadly conformable with bedding in the sedimentary rocks, but at outcrop scale, these smaller intrusions were observed to terminate abruptly in the metasedimentary rocks, and to be associated with ductile deformation in the country rocks. Some intrude the Upper Sill (Fig. 2b, c) while others are cut by this sill, indicating that there were several generations of minor intrusions.

On the Skerries, the Upper Sill was not found, but a $3 \mathrm{~m}$ thick dolerite sill was located and sampled no more than $4 \mathrm{~m}$ above the Main Sill (ANT24 \& ANT28, Fig. 1b). Metasediments are present on this side of the sill, but their thickness is less than $5 \mathrm{~m}$.

\section{Mineralogy}

\section{5.a. Igneous rocks}

All of the igneous rocks sampled for this study are either dolerites or gabbros (Fig. 4). The Main Sill is divided into the three divisions described by Hawkes $\&$ Wilson (1975). The upper division is a surprisingly thin chill zone in contact with the metasedimentary rocks (Fig. 2f). It has an aphanitic texture only in the uppermost few centimetres, then grades rapidly down into fine- to relatively coarse-grained dolerite. At a distance of only $20 \mathrm{~cm}$ from the contact, the average grain size is about 0.5 to $1 \mathrm{~mm}$ and the olivine content is only about $4 \%$. The middle division is equigranular gabbro with a grain size ranging from about 1 to $5 \mathrm{~mm}$. It is composed of plagioclase, clinopyroxene, about $5 \%$ olivine altered to iddingsite and accessory oxides. The lower division has a coarser texture (grain size can 

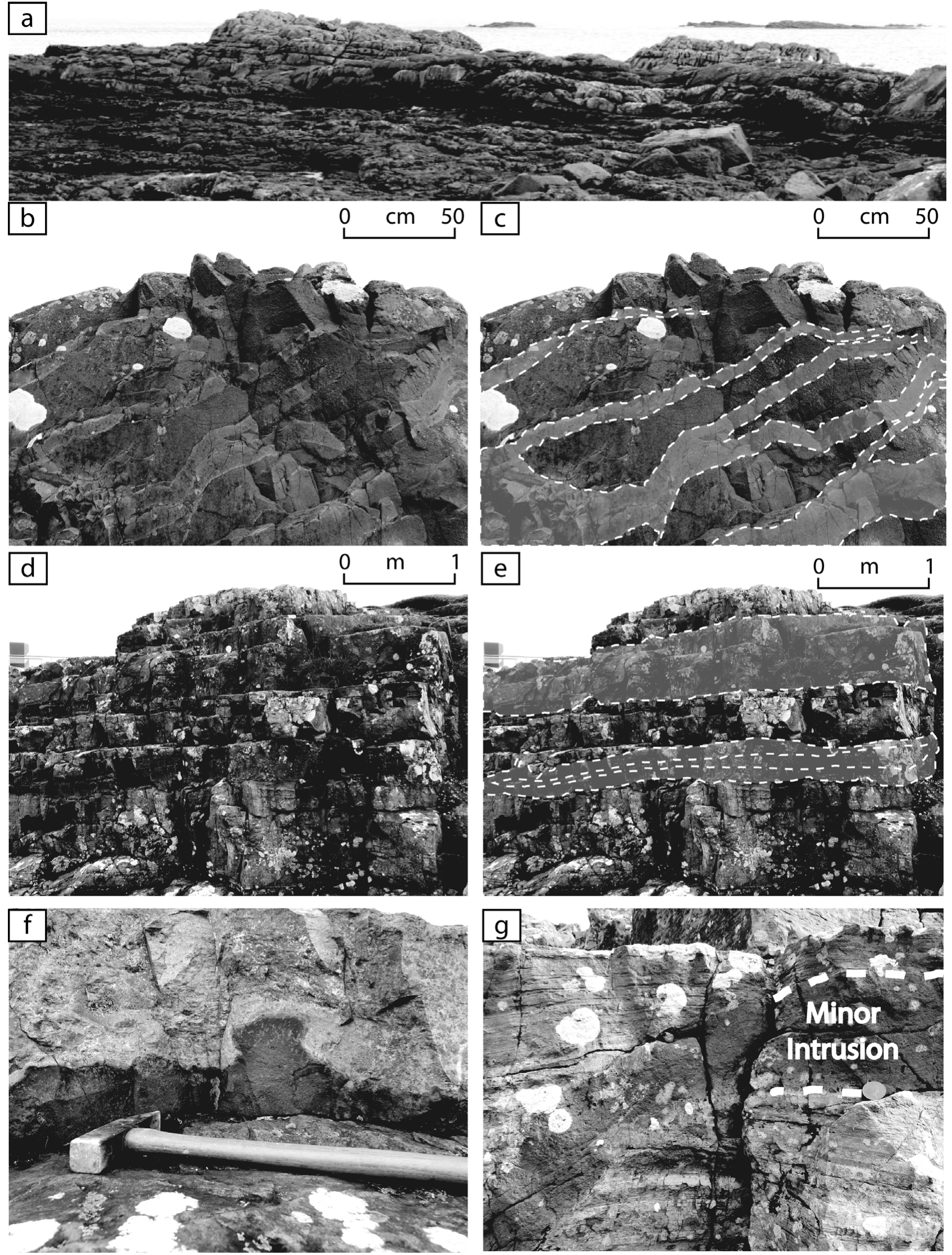

Figure 2. (a) General view of gabbros of the Portrush Sill with the N-dipping main outcrop in the foreground and the S-dipping outcrops of the Skerries in the background. The change in dip reveals the saucer- or lopolithic shape of the intrusion. (b) Dolerite of the Upper Sill cut by Minor Intrusions. (c) Same picture as (b) with Minor Intrusions outlined. (d) Minor Intrusions cutting through metasediments immediately below the Upper Sill. Those are concordant with the sediment layering. (e) Same picture as (d) with Minor Intrusions outlined. (f) The irregular fine-grained upper chilled margin of the Main Sill. (g) One of the Minor Intrusions cutting discordantly through metasediment: note the absence of a chilled margin at the edge of the intrusion. 


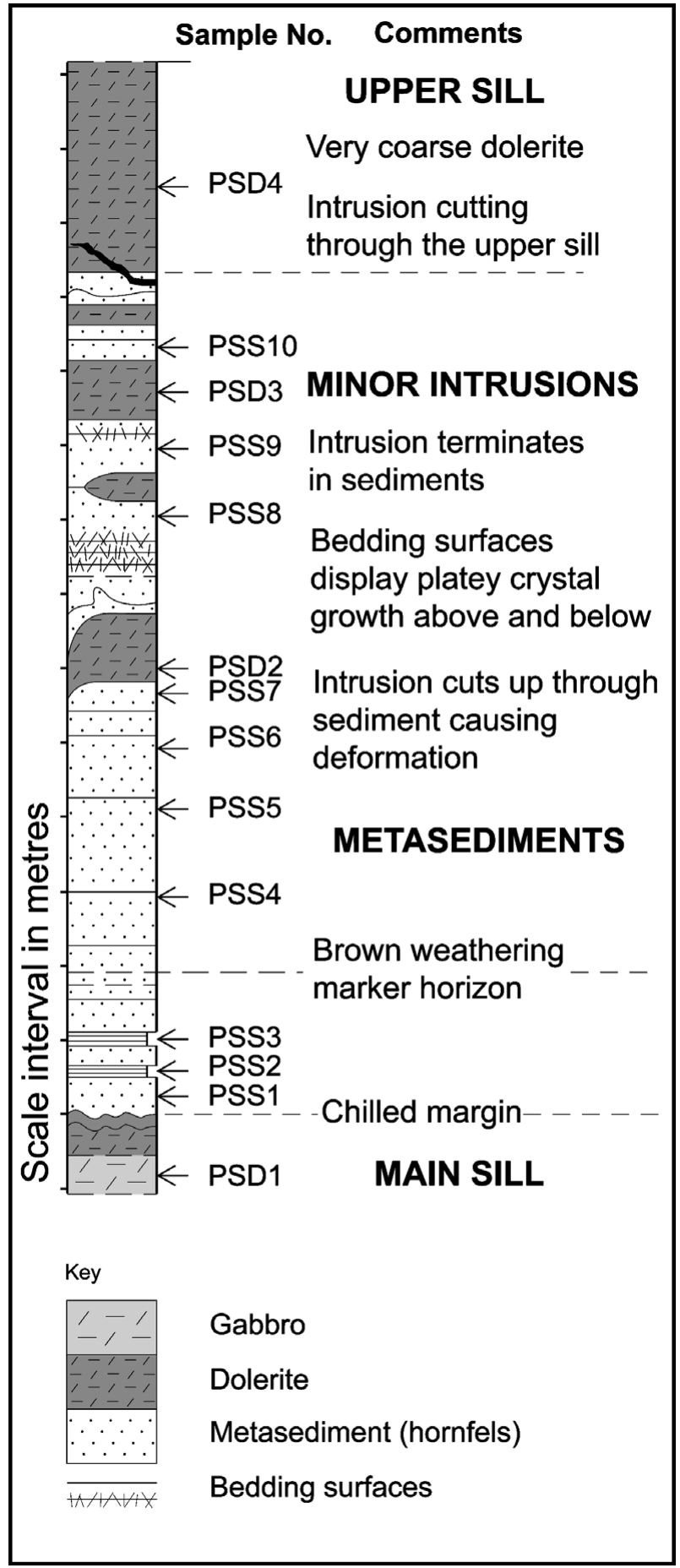

Figure 3. Log of metasediments between the Main Sill and Upper Sill showing sample sites. This diagram outlines the various relationships between the sedimentary and magmatic units.

reach centimetre scale) and higher olivine content at 10 to $15 \%$.

The Upper Sill is a medium-grained dolerite (grain size $<3 \mathrm{~mm}$ ) that contains a higher proportion of olivine $(\sim 20 \%)$ and clinopyroxene $(>30 \%)$ than the Main Sill. The Minor Intrusions have similar parageneses, but are more heterogeneous and are even finer grained $(<0.5 \mathrm{~mm})$. They have distinct but narrow chilled margins and often show fine banding parallel to their borders (Fig. 2g).
The $3 \mathrm{~m}$ thick intrusion overlying the Main Sill on the Skerries shows a pronounced variation in grain size, from an aphanitic chill zone at the upper contact through to medium-grained dolerite at a distance of $1.5 \mathrm{~m}$ from the contact. The olivine content is similar to that observed on the mainland at about $5 \%$. The contact chill zone is marked by pronounced alteration of the magmatic texture of the rock and by complete transformation of olivine to iddingsite.

\section{5.b. Sedimentary rocks}

The sedimentary rocks that host the Portrush Sill, at the Portrush waterfront and on the Skerries, consist entirely of mud- and siltstones that have been metamorphosed to hard, beige to grey hornfels (Fig. 2d, g). These hornfels have a quartzo-feldspathic matrix within which bioclasts can be recognized, including sponges with siliceous spicules, as well as Richardson's famous ammonites. No analyses were conducted on the protolith, which has been removed by erosion in the study area.

The hornfels are persistently fine grained $(<10 \mu \mathrm{m}$ to $<100 \mu \mathrm{m}$ ), which precludes positive identification of most mineral phases under the petrographic microscope. However, a combination of X-ray diffraction and microprobe analyses reveals that clay minerals are absent, having been replaced by an assemblage that includes potassium feldspar and plagioclase, $\mathrm{Mg}-\mathrm{Al}$ spinel and cordierite (Fig. 4). In samples taken next to the Minor Intrusions (PSS8 to PSS10, see Fig. 1c), orthopyroxene occurs as small equant grains scattered throughout the rock. In one sample (PSS8), a reaction rim of orthopyroxene surrounds a grain identified by microprobe analysis as the amphibole gedrite (Fig. 5). Finally, analyses of remnant magnetization by zero field cooling show the presence of two facies along the profile. The first is located in its central part (PSS2 to PSS6), far from all intrusion units, and contains both pyrrhotite and magnetite (Fig. 6a). The second comprises samples taken closer to the Minor Intrusions and is characterized by an absence of magnetite (PSS7 to PSS10) (Fig. 6b).

\section{Metamorphic conditions}

The metamorphic assemblages identified in the hornfels are consistent with metamorphism at low pressures and high temperatures, as we would expect in an aureole surrounding a shallow-level mafic intrusion. The presence of cordierite and spinel in all samples implies temperatures greater than $500^{\circ} \mathrm{C}$ across the entire profile. In the same way, pyrrhotite is ubiquitous; its appearance corresponds to the destabilization of pyrite, a reaction that occurs at temperatures above $523{ }^{\circ} \mathrm{C}$ (Scott, 1974). In sample PSS8, orthopyroxene forms at the expense of gedrite (Fig. 5):

gedrite + cordierite + quartz $\rightarrow$ orthopyroxene + cordierite 
MAGMATIC SAMPLES

\begin{tabular}{|c|c|c|c|c|c|c|c|}
\hline \multirow{2}{*}{ Sample } & \multirow{2}{*}{ Description } & \multicolumn{3}{|c|}{ Location } & \multirow{3}{*}{ Grain size } & \multirow{2}{*}{\multicolumn{2}{|c|}{ Mineralogy }} \\
\hline & & \multirow{3}{*}{$\begin{array}{c}\text { Site } \\
\text { Portrush }\end{array}$} & \multicolumn{2}{|c|}{ Irish Grid coordinates } & & & \\
\hline \multicolumn{2}{|c|}{ Main Sill } & & & & & & \\
\hline ANT34 & Gabbro & & 285434 & 441380 & $\begin{array}{c}\text { Coarse } \\
(0.5 \text { to } 1 \mathrm{~mm})\end{array}$ & $\begin{array}{l}\text { PI }(60-70 \%) \\
\text { Ox }\end{array}$ & $\begin{array}{l}\text { Ol }(\sim 4 \%) \\
\text { Cpx }(10-20 \%)\end{array}$ \\
\hline ANT35 & Gabbro & Portrush & 285434 & 441380 & $\begin{array}{l}\text { Coarse } \\
(1 \text { to } 5 \mathrm{~mm})\end{array}$ & $\begin{array}{l}\text { Pl }(60-70 \%) \\
\text { Ox }\end{array}$ & $\begin{array}{l}\text { OI }(\sim 5 \%) \\
\text { Cpx }(10-15 \%)\end{array}$ \\
\hline ANT36 & Gabbro & Portrush & 285434 & 441380 & $\begin{array}{c}\text { Coarse } \\
(1 \mathrm{~mm} \text { to } 1 \mathrm{~cm})\end{array}$ & $\begin{array}{l}\text { PI }(60-70 \%) \\
\text { Ox }\end{array}$ & $\begin{array}{l}\text { OI }(10-15 \%) \\
\text { Cpx }(15-20 \%)\end{array}$ \\
\hline \multicolumn{2}{|c|}{ Minor Intrusions } & & & & \multirow{6}{*}{$\begin{array}{c}\text { Fine } \\
(<0.5 \mathrm{~mm})\end{array}$} & \multirow{6}{*}{\multicolumn{2}{|c|}{$\begin{array}{c}\mathbf{P l}, \mathbf{C p x}, \mathbf{O I}, \mathbf{O x} \\
\text { in variable concentrations }\end{array}$}} \\
\hline PSD1 & Dolerite & Portrush & 285651 & 441199 & & & \\
\hline PSD2 & Dolerite & Portrush & 285771 & 440993 & & & \\
\hline PSD3 & Dolerite & Portrush & 285777 & 440987 & & & \\
\hline ANT24 & Dolerite & Skerries & 287466 & 442724 & & & \\
\hline ANT28 & Dolerite & Skerries & 287466 & 442724 & & & \\
\hline \multicolumn{2}{|c|}{ Upper Sill } & & & & & & \\
\hline PSD4 & Dolerite & Portrush & 285804 & 440967 & $\underset{(<3 \mathrm{~mm})}{\text { Medium }}$ & $\begin{array}{l}\mathbf{P l}(50-60 \%) \\
\mathbf{O x}\end{array}$ & $\begin{array}{c}\text { Ol }(\sim 20 \%) \\
\text { Cpx }(>30 \%)\end{array}$ \\
\hline
\end{tabular}

SEDIMENTARY SAMPLES

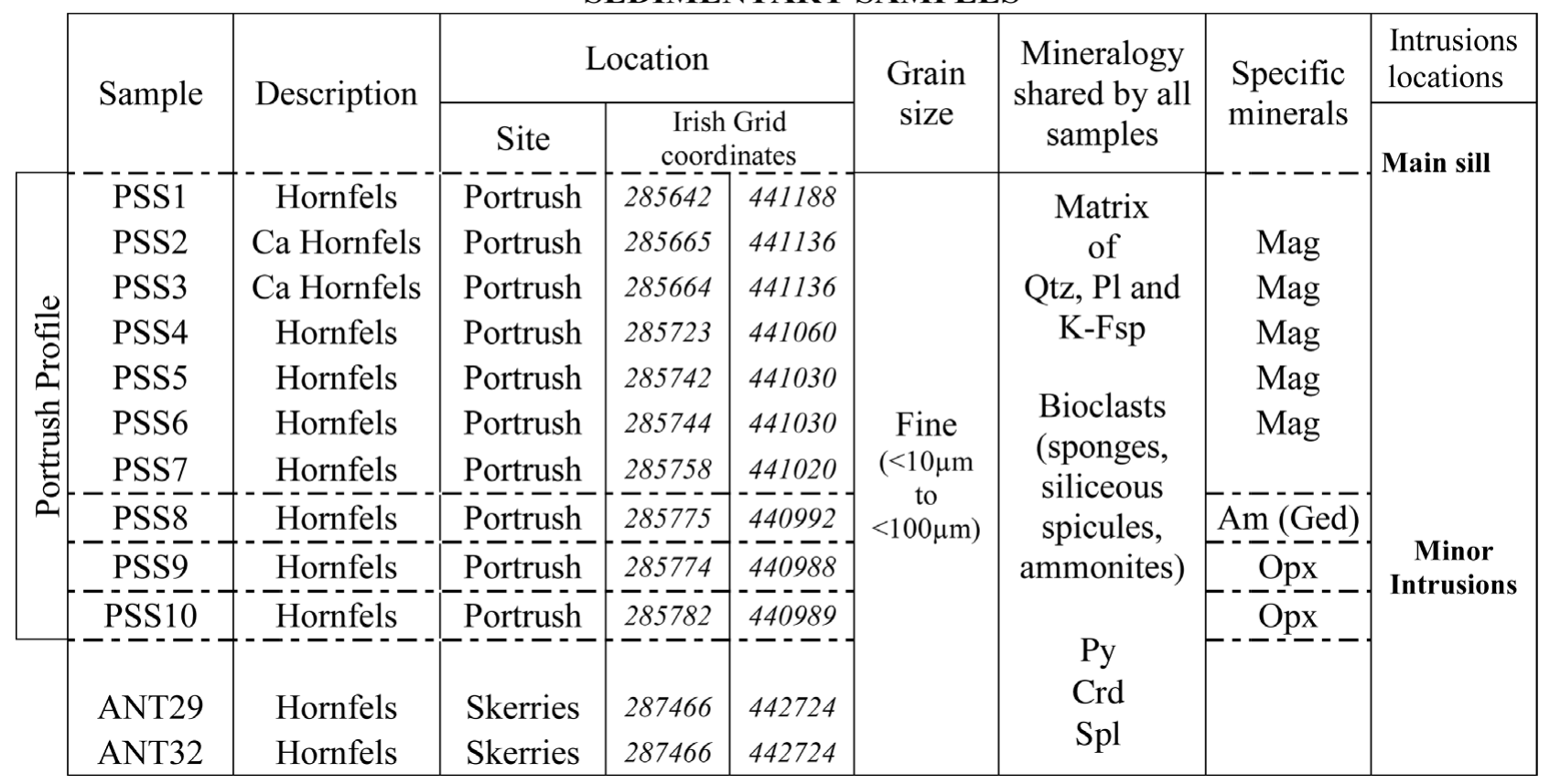

Figure 4. Sample descriptions with their localization (GPS coordinates from Irish grid reference), the rock type and their mineralogy. Minerals are named as follows: Pl - plagioclase; Ol - olivine; Cpx - clinopyroxene; Opx - orthopyroxene; Ox - oxides; Qtz - quartz; Fsp - feldspar; Am - amphibole; Ged - gedrite; Mag - magnetite: Crd - cordierite; Spl - spinel. The sedimentary part of the table includes horizontal dotted lines showing localization of magmatic intrusions along the Portrush profile.

To destabilize and transform the amphibole to pyroxene, the temperature must exceed $660^{\circ} \mathrm{C}$ (Winkler, 1965; Akella \& Winkler, 1966). These observations show that the whole profile has been subjected to thermal metamorphism at temperatures greater than $523{ }^{\circ} \mathrm{C}$, and that the upper part was hotter with temperatures in excess of $660^{\circ} \mathrm{C}$. The metamorphism is thus near constant across the $10 \mathrm{~m}$ of the aureole, with a slight maximum near the Minor Intrusions and far from the contact with the Main Sill.

\section{Geochemistry}

7.a. Igneous rocks

Major- and trace-element analyses of the magmatic rocks were conducted for the three units: the Main Sill, the Minor Intrusions and the Upper Sill. The compositions are similar to those of tholeiitic continental flood basalts: silica concentrations vary between 47 and $53 \mathrm{wt} \%$, and $\mathrm{CaO}$ remains higher than the sum of $\mathrm{Na}_{2} \mathrm{O}$ and $\mathrm{K}_{2} \mathrm{O}$. All the compositions are relatively evolved, 


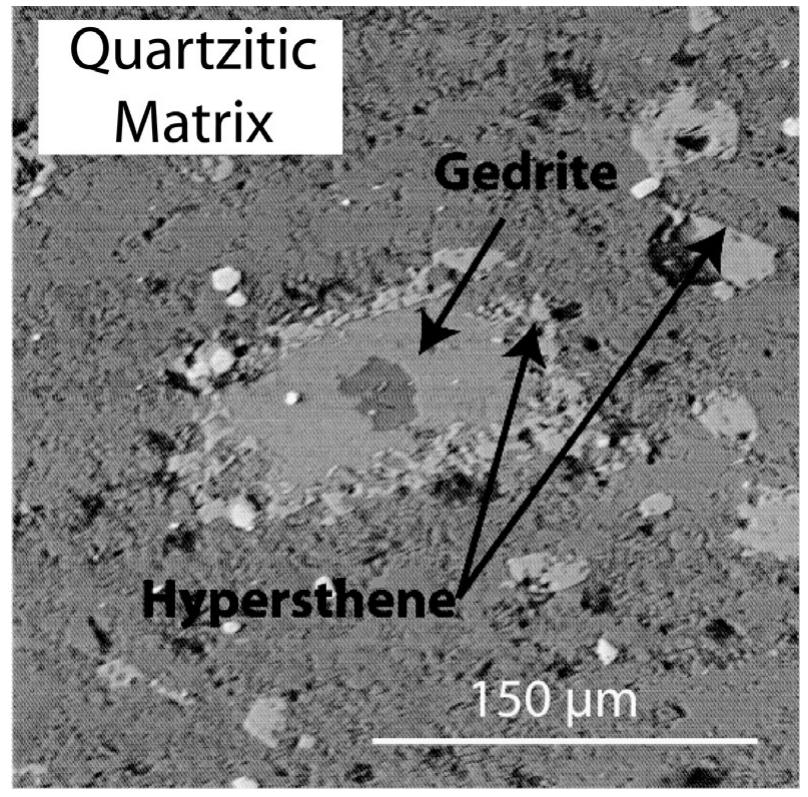

Figure 5. Back-scattered electron image of hornfels sample PSS8 showing metamorphic reaction rim of hypersthene on gedrite, identified by microprobe analyses. with $\mathrm{MgO}$ contents and $\mathrm{Mg}$ no. around $5 \mathrm{wt} \%$ and 0.27 , respectively, for the Main Sill, and around $8 \mathrm{wt} \%$ and 0.37 to 0.48 for the Minor Intrusions and the Upper Sill. Only the sample ANT24, taken at the contact with metasediments on the Skerries, is different from other Minor Intrusions with a $\mathrm{MgO}$ content of $5.67 \mathrm{wt} \%$ (Table 1).

The trace-element analyses, when plotted in multielement diagrams (Fig. 7a), reveal strong enrichment of the more incompatible elements coupled with pronounced negative niobium $(\mathrm{Nb})$ and tantalum (Ta) anomalies. These features are typical of basalts in continental large igneous provinces. All three lithological divisions of the Main Sill, as recognized by Hawkes \& Wilson (1975), have similar compositions and, unlike the Minor Intrusions and the Upper Sill, have positive barium $(\mathrm{Ba})$ anomalies. In contrast, the Minor Intrusions and the Upper Sill are very similar and show large positive lithium (Li) and lead $(\mathrm{Pb})$ anomalies, the amplitude of which varies from sample to sample. Finally, overall concentrations of trace elements in the Main Sill are distinctly higher than in the Minor Intrusions and in the Upper Sill (Table 2), despite its lower olivine content and its lower $\mathrm{Mg}$ no.

Table 1. Major element abundances for sedimentary and magmatic samples from Portrush and Skerries

\begin{tabular}{|c|c|c|c|c|c|c|c|c|c|c|c|c|c|c|}
\hline \multicolumn{15}{|c|}{ Sedimentary samples } \\
\hline \multirow{3}{*}{$\begin{array}{l}\text { (wt } \\
\%)\end{array}$} & \multicolumn{10}{|c|}{ PORTRUSH } & \multicolumn{2}{|c|}{ SKERRIES } & \multicolumn{2}{|c|}{ Standard* } \\
\hline & PSS & PSS & PSS & PSS & PSS & PSS & PSS & PSS & PSS & PSS & ANT & ANT & JSd1 & JSd1 \\
\hline & 1 & 2 & 3 & 4 & 5 & 6 & 7 & 8 & 9 & 10 & 29 & 32 & Measured & Theoretic \\
\hline $\mathrm{SiO}_{2}$ & 55.00 & 42.04 & 39.46 & 60.68 & 56.37 & 56.55 & 52.87 & 53.32 & 55.25 & 54.97 & 55.22 & 55.45 & 65.05 & 66.55 \\
\hline $\mathrm{TiO}_{2}$ & 0.99 & 0.71 & 0.74 & 0.96 & 0.92 & 1.00 & 0.97 & 0.98 & 1.00 & 0.99 & 0.96 & 1.03 & 0.61 & 0.643 \\
\hline $\mathrm{Al}_{2} \mathrm{O}_{3}$ & 21.59 & 15.32 & 17.72 & 17.78 & 18.45 & 20.26 & 21.48 & 21.50 & 18.82 & 21.75 & 17.00 & 12.53 & 14.44 & 14.65 \\
\hline $\mathrm{Fe}_{2} \mathrm{O}_{3 \text { tot }}$ & 6.97 & 8.46 & 7.25 & 6.05 & 6.57 & 6.45 & 7.72 & 7.20 & 7.14 & 8.01 & 6.97 & 6.39 & 4.91 & 5.02 \\
\hline $\mathrm{MnO}$ & 0.05 & 0.21 & 0.22 & 0.05 & 0.06 & 0.05 & 0.08 & 0.06 & 0.06 & 0.07 & 0.06 & 0.04 & 0.09 & 0.0924 \\
\hline $\mathrm{MgO}$ & 2.82 & 3.51 & 2.57 & 2.17 & 2.55 & 2.62 & 3.52 & 2.94 & 2.51 & 2.93 & 2.49 & 1.94 & 1.78 & 1.813 \\
\hline $\mathrm{CaO}$ & 5.06 & 23.08 & 22.53 & 3.08 & 4.97 & 3.60 & 6.38 & 4.88 & 3.89 & 4.38 & 4.32 & 0.53 & 2.99 & 3.034 \\
\hline $\mathrm{Na}_{2} \mathrm{O}$ & 0.98 & 0.77 & 0.85 & 1.10 & 1.04 & 1.09 & 1.14 & 1.11 & 1.21 & 1.01 & 1.62 & 0.91 & 2.73 & 2.727 \\
\hline $\mathrm{K}_{2} \mathrm{O}$ & 3.35 & 0.64 & 2.58 & 3.05 & 3.05 & 3.20 & 3.41 & 3.77 & 3.27 & 3.43 & 2.15 & 2.59 & 2.18 & 2.183 \\
\hline $\mathrm{P}_{2} \mathrm{O}_{5}$ & $<\mathrm{LD}$ & $<\mathrm{LD}$ & $<\mathrm{LD}$ & $<\mathrm{LD}$ & $<\mathrm{LD}$ & 0.15 & $<\mathrm{LD}$ & 0.13 & $<\mathrm{LD}$ & $<\mathrm{LD}$ & $<\mathrm{LD}$ & $<\mathrm{LD}$ & $<\mathrm{LD}$ & 0.122 \\
\hline LOI & 0.89 & 2.15 & 4.27 & 5.10 & 1.76 & 3.18 & 0.96 & 1.67 & 3.15 & 1.66 & 0.98 & 1.90 & no meas. & no meas. \\
\hline Total & 97.69 & 96.89 & 98.17 & 100.04 & 95.74 & 98.15 & 98.53 & 97.57 & 96.29 & 99.20 & 91.77 & 83.31 & 94.78 & 96.83 \\
\hline \multicolumn{15}{|c|}{ Magmatic samples } \\
\hline \multirow{4}{*}{$\begin{array}{l}\text { (wt } \\
\%)\end{array}$} & \multicolumn{4}{|c|}{ Main Sill } & \multicolumn{6}{|c|}{ Minor Intrusions } & \multicolumn{2}{|c|}{ Upper Sill } & & \\
\hline & \multicolumn{4}{|c|}{ PORTRUSH } & \multicolumn{3}{|c|}{ PORTRUSH } & \multicolumn{3}{|c|}{ SKERRIES } & \multicolumn{2}{|c|}{ PORTRUSH } & \multicolumn{2}{|c|}{ Standard* } \\
\hline & ANT & ANT & & INT & PSD & PSD & PSD & ANT & Al & NT & & $\mathrm{SD}$ & BEN & BEN \\
\hline & 34 & 35 & & 36 & 1 & 2 & 3 & 24 & 2 & 8 & & 4 & Measured & Theoretic \\
\hline $\mathrm{SiO}_{2}$ & 50.41 & 48.02 & & 8.64 & 45.76 & 46.02 & 46.68 & 50.42 & 46. & .94 & & 5.68 & 38.28 & 38.2 \\
\hline $\mathrm{TiO}_{2}$ & 1.07 & 1.46 & & .46 & 0.94 & 0.91 & 0.78 & 0.94 & 0.8 & 87 & & .83 & 2.67 & 2.61 \\
\hline $\mathrm{Al}_{2} \mathrm{O}_{3}$ & 13.14 & 12.77 & & 2.68 & 15.72 & 15.89 & 16.23 & 18.86 & 16. & .25 & & 6.14 & 10.02 & 10.07 \\
\hline $\mathrm{Fe}_{2} \mathrm{O}_{3 \text { tot }}$ & 11.16 & 13.32 & & 4.04 & 11.19 & 11.48 & 10.48 & 9.49 & 10 & .95 & & 69 & 12.87 & 12.84 \\
\hline $\mathrm{MnO}$ & 0.20 & 0.23 & & .23 & 0.18 & 0.19 & 0.18 & 0.13 & 0 . & 18 & & .16 & 0.20 & 0.2 \\
\hline $\mathrm{MgO}$ & 4.40 & 5.00 & & 5.08 & 7.96 & 8.20 & 8.76 & 5.67 & 8. & 46 & & 62 & 13.11 & 13.15 \\
\hline $\mathrm{CaO}$ & 8.13 & 9.61 & & 9.04 & 10.64 & 11.70 & 11.87 & 8.35 & 11. & .28 & & .21 & 13.95 & 13.87 \\
\hline $\mathrm{Na}_{2} \mathrm{O}$ & 3.24 & 3.07 & & .21 & 2.52 & 2.09 & 2.10 & 1.88 & 2. & 47 & & .08 & 3.23 & 3.18 \\
\hline $\mathrm{K}_{2} \mathrm{O}$ & 2.02 & 1.44 & & .76 & 0.32 & 0.26 & 0.27 & 1.40 & 0. & 34 & & .29 & 1.39 & 1.39 \\
\hline $\mathrm{P}_{2} \mathrm{O}_{5}$ & 0.15 & 0.12 & & KLD & $<\mathrm{LD}$ & 0.14 & $<\mathrm{LD}$ & 0.27 & $<\mathrm{I}$ & LD & & LD & 1.17 & 1.05 \\
\hline LOI & 2.73 & 1.64 & & 2.08 & 2.40 & 0.25 & 0.26 & 0.70 & 1.2 & 29 & & .98 & no mes. & 2.45 \\
\hline Total & 98.04 & 99.22 & & .55 & 97.61 & 97.12 & 97.61 & 98.09 & 99. & & 96. & 68 & 96.91 & no mes. \\
\hline Mg no. & 0.28 & 0.27 & & .27 & 0.42 & 0.42 & 0.46 & 0.37 & 0 & 44 & & .47 & 0.50 & 0.50 \\
\hline
\end{tabular}

Abbreviations: LD - limit of detection; LOI - loss on ignition

*BEN values from Govindaraju (1980); JSd1 values from Terashima et al. (1990). 
Table 2. Trace element abundances for sedimentary and magmatic samples from Portrush and Skerries

\begin{tabular}{|c|c|c|c|c|c|c|c|c|c|c|c|c|c|c|c|c|c|c|c|c|c|c|c|}
\hline \multicolumn{13}{|c|}{ SEDIMENTARY SAMPLES } & \multicolumn{11}{|c|}{ MAGMATIC SAMPLES } \\
\hline & & & & & & & & & & & \multirow{2}{*}{\multicolumn{2}{|c|}{ SKERRIES }} & \multirow{2}{*}{\multicolumn{3}{|c|}{$\begin{array}{c}\text { Main Sill } \\
\text { PORTRUSH }\end{array}$}} & \multicolumn{5}{|c|}{ Minor Intrusions } & \multirow{3}{*}{$\frac{\frac{\text { Upper Sill }}{\text { PORTRUSH }}}{\text { PSD } 4}$} & \multicolumn{2}{|c|}{ Standard* } \\
\hline \multicolumn{11}{|c|}{ PORTRUSH } & & & & & & & ORTRUS & & SKERI & RIES & & BEN & BEN \\
\hline (ppm) & PSS 1 & PSS 2 & PSS 3 & PSS 4 & PSS 5 & PSS 6 & PSS 7 & PSS 8 & PSS 9 & PSS 10 & ANT 29 & ANT 32 & ANT 34 & ANT 35 & ANT 36 & PSD 1 & PSD 2 & PSD 3 & ANT 24 & ANT 28 & & Meas. & Th. \\
\hline Cs & 9.21 & 7.44 & 6.77 & 4.2 & 5.72 & 6.77 & 7.18 & 6.83 & 6.2 & 7.49 & 6.45 & 9.31 & 0.197 & 0.214 & 0.29 & 0.307 & 0.213 & 0.149 & 3.44 & 0.118 & 0.13 & 0.743 & 0.74 \\
\hline $\mathrm{Rb}$ & 136 & 24.7 & 108 & 125 & 133 & 121 & 104 & 65.6 & 134 & 142 & 115 & 104 & 20 & 18.2 & 22.5 & 6.98 & 3.73 & 4.21 & 64.1 & 6.72 & 4.06 & 47.8 & 49.6 \\
\hline $\mathrm{Ba}$ & 305 & 229 & 232 & 362 & 357 & 307 & 248 & 181 & 421 & 321 & 237 & 224 & 737 & 508 & 562 & 127 & 114 & 101 & 182 & 117 & 98.7 & 1045 & 1056 \\
\hline Th & 15.3 & 13.2 & 12.2 & 15 & 14.5 & 14.4 & 14.9 & 13.8 & 14.9 & 15.1 & 15.7 & 13.4 & 0.966 & 0.586 & 0.578 & 0.504 & 0.414 & 0.449 & 7.91 & 0.473 & 0.317 & 10.6 & 10.9 \\
\hline & 2.92 & 3.86 & 4.39 & 3.5 & 3.28 & 3.39 & 3.35 & 2.61 & 3.28 & 2.89 & 2.62 & 3.07 & 0.244 & 0.178 & 0.224 & 0.107 & 0.102 & 0.109 & 1.31 & 0.127 & 0.0797 & 2.42 & 2.46 \\
\hline $\mathrm{Nb}$ & 21.9 & 16.6 & 15.5 & 20.7 & 20.4 & 21.3 & 20.4 & 20.3 & 21.6 & 21.4 & 20.2 & 23.5 & 3.49 & 2.87 & 2.76 & 2.76 & 2.27 & 2.26 & 11 & 2.63 & 1.74 & 117 & 101 \\
\hline $\mathrm{Ta}$ & 1.43 & 1.09 & 1.05 & 1.31 & 1.29 & 1.35 & 1.32 & 1.36 & 1.41 & 1.4 & 1.32 & 1.55 & 0.175 & 0.136 & 0.133 & 0.151 & 0.132 & 0.126 & 0.768 & 0.141 & 0.104 & 5.58 & 6.03 \\
\hline $\mathrm{La}$ & 44.3 & 49.9 & 43.9 & 44.9 & 43.9 & 42 & 43.4 & 40.1 & 43.6 & 43 & 45.3 & 40.9 & 11.6 & 8.25 & 8.2 & 4.59 & 3.93 & 3.88 & 27.4 & 4.21 & 2.99 & 80 & 82.7 \\
\hline $\mathrm{Ce}$ & 95.9 & 111 & 88.4 & 101 & 98.2 & 94.4 & 96.3 & 92.8 & 97.8 & 96 & 91.9 & 82.5 & 26.9 & 19 & 19.5 & 10.9 & 9.56 & 9.23 & 57.5 & 10.1 & 7.12 & 147 & 154 \\
\hline Pr & 10.7 & 12.3 & 10.3 & 11.3 & 11.3 & 10.4 & 11.2 & 10.3 & 10.7 & 10.5 & 11.1 & 10 & 3.88 & 2.82 & 2.78 & 1.69 & 1.53 & 1.42 & 7.11 & 1.59 & 1.15 & 17.1 & 17.3 \\
\hline $\mathrm{Pb}$ & 33.3 & 1.58 & 28.4 & 22.4 & 24.8 & 28.2 & 23.1 & 12.4 & 37.5 & 22.2 & 19.4 & 26.6 & 3.36 & 2.18 & 2.47 & 1.11 & 1.14 & 6.66 & 13 & 1.24 & 1.5 & 5.74 & 3.97 \\
\hline $\mathrm{Nd}$ & 37.9 & 45.7 & 38.1 & 40.2 & 40.4 & 37.4 & 40.3 & 39.9 & 39.9 & 38.9 & 40.9 & 37 & 17.5 & 13.5 & 13.5 & 8.45 & 7.81 & 7.13 & 27.7 & 7.8 & 5.58 & 63.4 & 67.1 \\
\hline $\mathrm{Sr}$ & 295 & 528 & 424 & 217 & 266 & 248 & 309 & 296 & 261 & 290 & 284 & 132 & 277 & 284 & 260 & 176 & 169 & 159 & 229 & 186 & 167 & 1374 & 1420 \\
\hline $\mathrm{Sm}$ & 7.07 & 8.68 & 7.42 & 7.64 & 7.23 & 6.84 & 7.78 & 7.66 & 7.4 & 7.57 & 7.74 & 7.05 & 4.91 & 3.94 & 3.87 & 2.67 & 2.52 & 2.24 & 5.86 & 2.5 & 1.86 & 12 & 12.4 \\
\hline $\mathrm{Zr}$ & 200 & 177 & 128 & 321 & 245 & 221 & 176 & 179 & 255 & 208 & 184 & 189 & 108 & 71.3 & 70.8 & 30.3 & 48.8 & 46.2 & 67.9 & 52.5 & 37.9 & 286 & 273 \\
\hline $\mathrm{Hf}$ & 4.87 & 4.37 & 3.24 & 7.87 & 6.15 & 5.34 & 4.51 & 4.56 & 6.26 & 5.05 & 4.84 & 4.89 & 2.86 & 1.93 & 1.9 & 1.04 & 1.41 & 1.31 & 2.23 & 1.48 & 1.09 & 5.46 & 5.71 \\
\hline $\mathrm{Eu}$ & 1.45 & 1.71 & 1.51 & 1.43 & 1.47 & 1.42 & 1.58 & 1.59 & 1.46 & 1.5 & 1.55 & 1.49 & 1.77 & 1.48 & 1.45 & 0.993 & 0.997 & 0.886 & 1.43 & 0.964 & 0.783 & 3.38 & 3.91 \\
\hline Gd & 5.54 & 6.97 & 6.28 & 5.99 & 5.6 & 5.4 & 6.2 & 6. & 5. & & 6.1 & 5.8 & 6.41 & & 4.9 & 3.39 & 3.32 & 2.99 & 5.5 & 3.3 & 2.56 & 9.36 & 10.4 \\
\hline $\mathrm{Tb}$ & 0.874 & 1.1 & 0.959 & 0.932 & 0.921 & 0.872 & 0.991 & 0.984 & 0.921 & 0.952 & 0.99 & 0.96 & 1.19 & 0.96 & 0.931 & 0.651 & 0.641 & 0.577 & 0.911 & 0.614 & 0.485 & 1.26 & 1.35 \\
\hline Dy & 5.06 & 6.19 & 5.27 & 5.43 & 5.08 & 4.94 & 5.59 & 5.56 & 5.37 & & & 5.44 & 7.7 & & 6.05 & 4.14 & 4.11 & 3.82 & 5.54 & 4.01 & 3.2 & 6.11 & 6.53 \\
\hline Ho & 1.03 & 1.23 & 1.14 & 1.12 & 1.04 & 0.993 & 1.13 & 1.13 & 1.09 & 1.09 & 1.17 & 1.11 & 1.75 & 1.41 & 1.38 & 0.929 & 0.906 & 0.849 & 1.14 & 0.902 & 0.727 & 1.05 & 1.08 \\
\hline $\mathrm{Y}$ & 30.1 & 36 & 34.1 & 33.1 & 30.6 & 29.1 & 31.4 & 31.4 & 31 & 31.4 & 34.4 & 31.7 & 52.2 & 41.8 & 40.8 & 27.6 & 26.7 & 24.8 & 33.1 & 26.3 & 21.5 & 31 & 29.6 \\
\hline Er & 2.96 & 3.43 & 3.16 & 3.23 & 3.05 & 2.98 & 3.22 & 3.27 & 3.14 & 3.09 & 3.37 & 3.28 & 5.23 & 4.24 & 4.06 & 2.83 & 2.73 & 2.5 & 3.2 & 2.65 & 2.23 & 2.51 & 2.71 \\
\hline $\mathrm{Li}$ & 113 & 32.5 & 72.1 & 127 & 119 & 169 & 129 & 127 & 119 & 114 & 64.8 & 119 & 7.97 & 8.84 & 8.31 & 14.1 & 85.2 & 56.8 & 44.4 & 8.7 & 7.18 & 12.7 & 12.5 \\
\hline $\mathrm{Yb}$ & 2.88 & 3.09 & 2.92 & 3.13 & 2.92 & 2.93 & 3.04 & 3.06 & 2.99 & 2.98 & 3.08 & 3.08 & 5.13 & 3.97 & 3.86 & 2.56 & 2.51 & 2.34 & 2.9 & 2.53 & 2.14 & 1.73 & 1.77 \\
\hline $\mathrm{Lu}$ & 0.444 & 0.452 & 0.423 & 0.485 & 0.441 & 0.436 & 0.461 & 0.462 & 0.465 & 0.463 & 0.484 & 0.461 & 0.791 & 0.603 & 0.581 & 0.39 & 0.388 & 0.358 & 0.427 & 0.389 & 0.318 & 0.244 & 0.25 \\
\hline $\mathrm{Ta} / \mathrm{N}$ & 0.07 & 0.07 & 0.07 & 0.06 & 0.06 & 0.06 & 0.06 & 0.07 & 0.07 & 0.07 & 0.07 & 0.07 & 0.05 & 0.05 & 0.05 & 0.05 & 0.06 & 0.06 & 0.07 & 0.05 & 0.06 & 0.05 & 0.06 \\
\hline $\mathrm{Li} / \mathrm{Yb}$ & 39.24 & 10.52 & 24.69 & 40.58 & 40.75 & 57.68 & 42.43 & 41.50 & 39.80 & 38.26 & 21.04 & 38.64 & 1.55 & 2.23 & 2.15 & 5.51 & 33.94 & 24.27 & 15.31 & 3.46 & 3.36 & 7.34 & 7.06 \\
\hline $\mathrm{Ba} / \mathrm{Th}$ & 19.93 & 17.35 & 19.02 & 24.13 & 24.62 & 21.32 & 16.64 & 13.12 & 28.26 & 21.26 & 15.10 & 16.72 & 762.94 & 866.89 & 972.32 & 251.98 & 275.36 & 224.94 & 23.01 & 247.36 & 311.36 & 98.58 & 96.88 \\
\hline
\end{tabular}

${ }^{*}$ BEN values from Govindaraju (1980). Meas. $=$ measured; Th. $=$ Theoretic. 

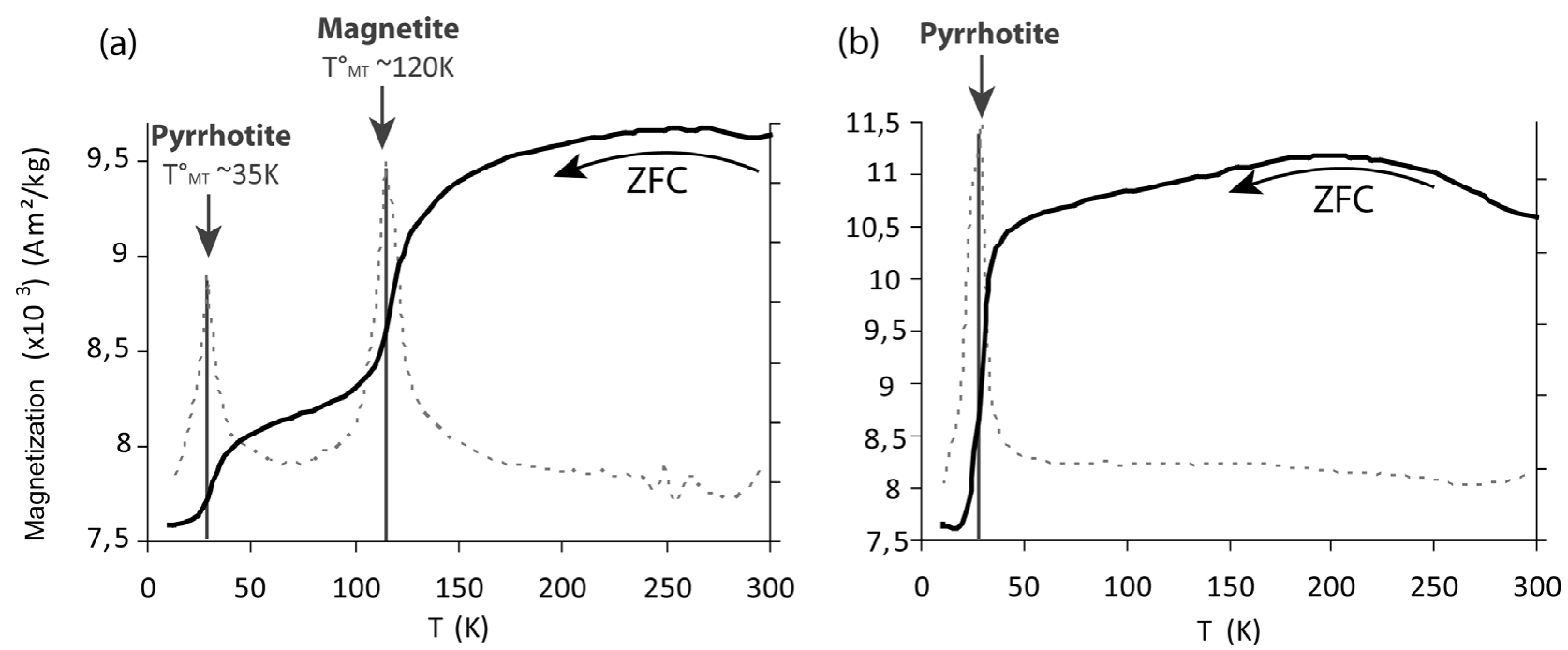

Figure 6. Zero field cooling curves for representative hornfels samples showing two mineral assemblages: (a) Type 1, from the centre of the sedimentary profile: magnetite and pyrrhotite; (b) Type 2, adjacent to a Minor Intrusion: pyrrhotite only. Temperatures are of magnetic transition and reveal the presence of either magnetite at $120 \mathrm{~K}$ or pyrrhotite at $35 \mathrm{~K}$.

Two igneous samples from the Minor Intrusion located just above the Main Sill on the Skerries have unusual compositions (Table 2). The first was collected less than $2 \mathrm{~cm}$ from the contact with sediment (ANT24), and the second at a depth of about $3 \mathrm{~m}$ within the sill (ANT28). The multi-element spectrum of the first sample has a sedimentary signal very like that of Portrush hornfels, whereas the second sample has a composition similar to those of the Minor Intrusions (Fig. 7b).

\section{7.b. Metasedimentary rocks}

Samples from the contact aureole have very uniform compositions (Table 1) with 52.87 to $60.68 \% \mathrm{SiO}_{2}$, 17.78 to $21.75 \% \mathrm{Al}_{2} \mathrm{O}_{3}, 2.17$ to $3.52 \mathrm{wt} \% \mathrm{MgO}$ and 3.05 to $3.77 \mathrm{wt} \% \mathrm{~K}_{2} \mathrm{O}$, with the exception of two samples from fine-grained beds located about $20 \mathrm{~cm}$ above the contact (PSS2 \& PSS3, see Figs 1c \& 3): they have much higher $\mathrm{CaO}$ contents $(\sim 23 \mathrm{wt} \%$, compared with the 3.08 to $6.38 \mathrm{wt} \%$ in the other samples), probably indicating that they originally contained a high carbonate content. The two samples taken on the Skerries (ANT29 \& ANT32) are similar to those from Portrush: this confirms that the same Lower Jurassic metasediments are present on both side of the Main Sill. As suggested by the petrography (Fig. 4), the chemical analyses show that the composition of the sedimentary rocks is nearly constant through the contact aureole.

The trace-element analyses are typical of postArchaean argillaceous sediments, being characterized by enrichment of the more incompatible elements and pronounced negative europium (Eu) anomalies (Table 2; Fig. 7a). The multi-element spectra show variable zircon $(\mathrm{Zr})$ and hafnium $(\mathrm{Hf})$ anomalies that can be attributed to variable contents of detrital zircon and other heavy minerals (Fig. 7a).
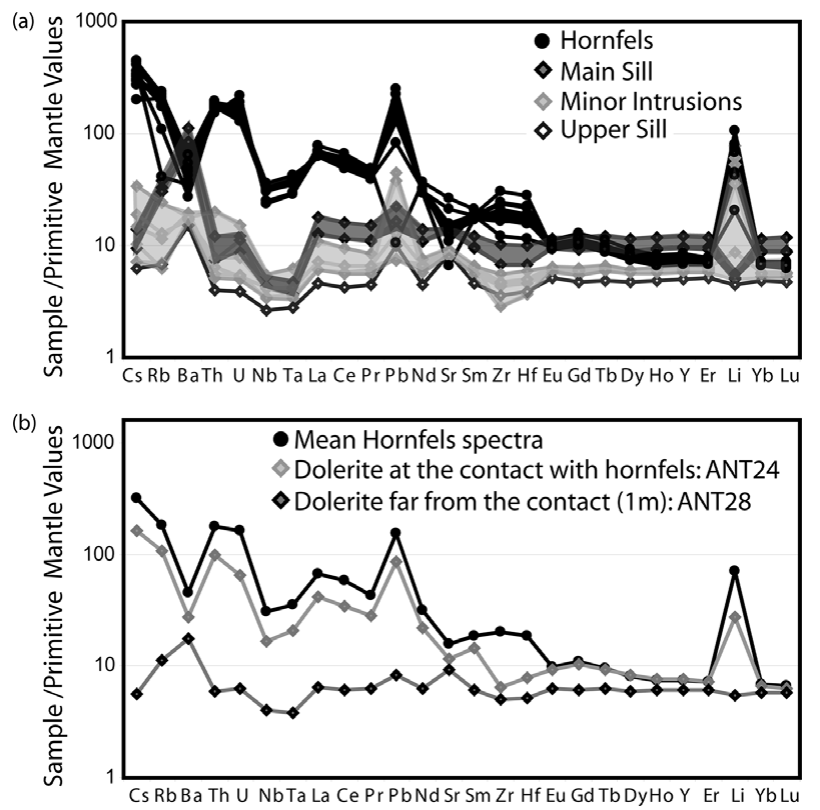

Figure 7. (a) Multi-element spectra of samples from the three dolerite or gabbro units of Portrush and associated hornfels normalized to the primitive mantle values of Hofmann (1988). $\mathrm{The} \mathrm{Nb}$ and $\mathrm{Ta}$ anomalies and the enrichment of all samples provide evidence of contamination by granitic crust. The Li, $\mathrm{Ba}$ and $\mathrm{Pb}$ anomalies give proof of shallower contamination. (b) Multi-element spectra showing the similarity between the mean hornfels composition and the composition of an adjacent Minor Intrusion (shallow dolerite, ANT 24), compared with a dolerite sampled far from the contact (deep dolerite, ANT 28). This correlation provides evidence of localized fluid circulation contamination.

When compared with the igneous rocks, we note that (1) negative anomalies of $\mathrm{Nb}$ and $\mathrm{Ta}$ are present in all the sedimentary rocks, (2) Ba forms very large negative anomalies in contrast with the positive anomalies 

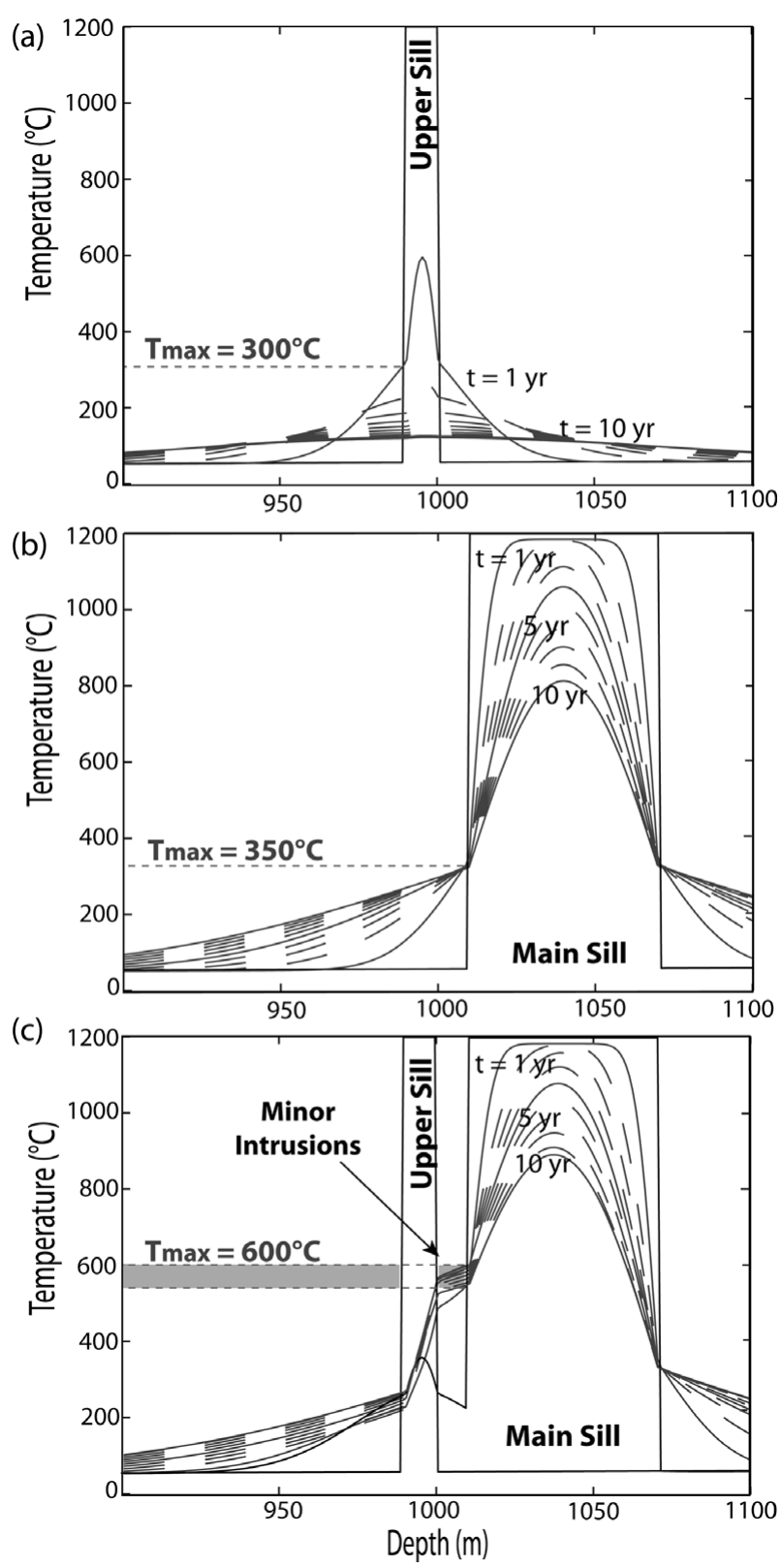

Figure 8. Numerical models of thermal diffusion following Fourier's heat equation. The whole system had a geothermal gradient of $30^{\circ} \mathrm{C} / \mathrm{km}$. The initial heat of the magma is fixed at $1200{ }^{\circ} \mathrm{C}$, the thermal diffusivity of the magma is $10^{-6} \mathrm{~m}^{2} / \mathrm{s}$, and the thermal diffusivity of the sediments is $10^{-5} \mathrm{~m}^{2} / \mathrm{s}$. Each sill was assumed to intrude at $950 \mathrm{~m}$ depth to prevent side effects. (a) The Upper Sill was modelled at a depth of $990 \mathrm{~m}$ with a thickness of $10 \mathrm{~m}$ : the highest temperature reached in the surrounding sediment is about $300^{\circ} \mathrm{C}$. (b) The Main Sill modelled at a depth of $1010 \mathrm{~m}$ with a thickness of $60 \mathrm{~m}$ : the highest temperature reached in the sediment is about $350^{\circ} \mathrm{C}$. (c) Both sills modelled at the same depths and the same thicknesses as before, but the Main Sill was emplaced one year after the Upper Sill. The thermal combination allows the sediments to reach a high temperature of almost $600{ }^{\circ} \mathrm{C}$. This model works for a lag time between intrusions of a few months to about five years.

displayed by most of the igneous rocks, and (3) positive $\mathrm{Pb}$ and $\mathrm{Li}$ anomalies are present in all metasediments and some igneous rocks.

\section{Discussion}

\section{8.a. Portrush igneous rocks and magma contamination} processes

We found that the structure of the Portrush Sill is somewhat more complex than described by Hawkes \& Wilson (1975). Of particular importance, in the context of this study, is the recognition of the Minor Intrusions and the Upper Sill in the sedimentary rocks overlying the Main Sill because, as discussed later, they perturbed the thermal profile developed in the aureole. In addition, they provide evidence for a complex history of interaction between mafic magma and crustal rocks.

The trace-element spectra, and especially the relative abundances of $\mathrm{Nb}, \mathrm{Ta}, \mathrm{Pb}, \mathrm{Ba}$ and $\mathrm{Li}$ (Fig. 7), provide evidence of two stages of contamination. The first is manifested in the overall enrichment in all samples of incompatible elements, accompanied by the development of negative $\mathrm{Nb}-\mathrm{Ta}$ anomalies. The isotope data of Barrat \& Nesbitt (1996) shows that igneous rocks of the Antrim Lava Group have variable ${ }^{87} \mathrm{Sr} /{ }^{86} \mathrm{Sr}$ and ${ }^{143} \mathrm{Nd} /{ }^{144} \mathrm{Nd}$ ratios, with $\varepsilon_{\mathrm{Sr}}(\mathrm{t})=-22$ to +107 and $\varepsilon_{\mathrm{Nd}}(\mathrm{t})=-11$ to +8.5 . Such geochemical characteristics are typical of continental flood basalts worldwide. Like Barrat \& Nesbitt (1996), we accept a contamination model involving interaction between magma and granitic rocks in a deep to mid-crustal magma chamber as an explanation for the overall geochemical features of the Antrim rocks.

The samples from the Minor Intrusions on the Skerries indicate that a second stage of magma contamination occurred at shallower depths. As shown in Figure $7 \mathrm{~b}$, positive anomalies in $\mathrm{Li}$ and $\mathrm{Pb}$ and a negative $\mathrm{Ba}$ anomaly relative to $\mathrm{Cs}, \mathrm{Rb}$ and $\mathrm{Th}$, which match those in the sedimentary rocks, are present in the sample from the margin of the intrusion (ANT24), but are poorly developed or absent in the sample from deeper in the intrusion (ANT28). This reveals localized contamination of the magma by sediments. As mentioned above, the magmatic minerals in the sample closest to the contact are replaced by secondary phases such as the iddingsite that replaces olivine, probably as a result of interaction with circulating aqueous fluids. The uptake of $\mathrm{Li}, \mathrm{Pb}$ and other alkali elements can therefore be attributed to the transfer of these soluble elements from sediment into the intrusion in the same type of fluid. In the same way, because the alkalis and alkaline earths are easily mobilized during fluid circulation, the sample at the contact became enriched in $\mathrm{K}_{2} \mathrm{O}$ and depleted in $\mathrm{FeO}$. The absence of $\mathrm{Li}, \mathrm{Pb}$ and $\mathrm{Ba}$ anomalies in rocks from deeper in the intrusion tells us that the assimilation of material from sedimentary host rocks is a relatively limited process, one that takes place at a post-magmatic stage and is restricted to the margins of Minor Intrusions.

\section{8.b. Portrush Sill: a special case of contact metamorphism}

The metamorphic aureole of the Portrush Sill is almost $10 \mathrm{~m}$ wide and extends from the upper contact of 
the Main Sill through to the base of the Upper Sill (Fig. 3). Rocks higher in the section have been removed by erosion. The mineralogical data show that high temperatures were reached in all parts of the aureole: the presence of cordierite and pyrrhotite throughout the aureole implies a minimum temperature of $500-520^{\circ} \mathrm{C}$ (Schreyer \& Yoder, 1964; Schreyer, 1965; Winkler, 1965), and in areas with multiple Minor Intrusions, i.e. in the upper part of the profile, the gedritehypersthene assemblage indicates temperatures over $660^{\circ} \mathrm{C}$. The entire sedimentary unit between the Main Sill and the Upper Sill has thus undergone intense thermal metamorphism. The temperature profile is quite different from what would be expected for one surrounding a single intrusion, in which the temperature should decline exponentially away from the intrusive contact. Another peculiarity is seen in the upper border zone of the Main Sill, which is unusually thin: the aphanitic chilled margin is only a few centimetres thick (Fig. 2f) and, at a depth only a metre into the sill, the rock is medium to coarse grained. Huppert \& Sparks (1989) and Holness \& Humphreys (2003) described how a chilled margin might form initially at the border of a sill, only to be resorbed by remelting or erosion caused by continued flowage of magma through the sill. We believe this process did not act during emplacement of the Portrush Sill for the following reasons. (1) The metamorphic profile inferred from mineral assemblages in the contact aureole records no decline in temperature away from the upper contact of the Main Sill. In the case of continuous magma flow associated with an eroded chilled margin, we would expect the higher temperatures at the edge of the intrusion; instead the highest temperatures are recorded between the small intrusions, some $8 \mathrm{~m}$ above the contact of the Main Sill. (2) In the metasediments of the aureole, textures and structures indicative of partial melting are absent while they are common features when sustained magma flows persist. (3) A chilled margin, although thin, is indeed present at the upper contact of the sill (Fig.3c). (4) Neither textures nor structures in the upper border zone of the sill have been found to sustain the hypothesis of continued flow and melt-back of the initial chilled margin: the texture changes progressively, from fine to coarse, away from the margin, and flow structures and textures indicative of remelting are absent. On the basis of these arguments, we propose that the Main Sill was emplaced essentially as a single pulse and that the thermal environment was anomalous - unusually hot - at the time of its intrusion.

To explain the high temperatures, we propose that the Upper Sill and the Minor Intrusions were emplaced into the sedimentary sequence shortly before the Main Sill. Given the similar chemical compositions of the smaller intrusions, it is probable that they had a common source and were emplaced in a brief interval of time; this is supported by the several generations of Minor Intrusions that cut through or are cut by the Upper Sill. The two sets of intrusions generated a thermal aureole that extended several metres down to the sedimentary sequence. The Main Sill then intruded into a pre-heated series of rocks, and the excess of temperature provided by the earlier intrusions prevented the formation of a thick chill zone.

We modelled the thermal evolution of the intrusions and the country rocks using a simple heat-flow equation (Fourier, 1822). To apply this equation requires knowledge of several critical parameters, many of which are poorly constrained. One crucial parameter is the thicknesses of both large sills, which we cannot determine because the upper contact of the Upper Sill and the lower contact of the Main Sill are not exposed. For the modelling we have assumed that the Upper Sill was $10 \mathrm{~m}$ thick and the Main Sill was $60 \mathrm{~m}$ thick. Other unknown parameters are the duration of magma flow through the sills, which, for the reasons given above, we believe was short, and the amount of time that elapsed between the emplacements of the two sills.

As shown in the Figure 8a and b, neither the Upper Sill alone nor the Main Sill alone can explain the high temperatures observed in the aureole: the highest temperatures they could produce are $300^{\circ} \mathrm{C}$ and $350{ }^{\circ} \mathrm{C}$, respectively, far less than the $550^{\circ} \mathrm{C}$ recorded in the metamorphic aureole. Coupling of the thermal effects of those two intrusions with transfer of heat from the Main Sill enhanced the temperature of rocks throughout the sedimentary interval, and the process was efficient enough to produce the temperatures of 500 to $600^{\circ} \mathrm{C}$.

The Minor Intrusions are not shown in this model, but they played a crucial role in the upper part of the temperature profile: as they were emplaced together with the Upper Sill, they are able to boost the temperature up to $660^{\circ} \mathrm{C}$ (compared to the $600{ }^{\circ} \mathrm{C}$ shown in the model) leading to the appearance of orthopyroxene in samples taken in this particular zone (PSS8, PSS9, PSS10).

Several other models were tested but not shown here, especially concerning variations in the lag time between both intrusions. What emerges from those models is that if the Main Sill intruded within a few years of the first intrusions $(<5$ years $)$, the temperatures are coherent with the mineralogy, but if the lag time is longer, temperatures are too low and inconsistent with the metamorphic assemblages.

The geochemical data, on the other hand, show differences in the levels of incompatible elements of the Main Sill and the overlying units (Table 2; Fig. 8a) and provide evidence that they are not related by simple fractional crystallization. The combination of low REE contents but high Mg no. of the Upper Sill and Minor Intrusions suggests they derive from a different parental magma than the Main Sill. The Main Sill is then not directly co-magmatic with those two units, and their close proximity - in space and in time - provides evidence of the complexity of this magmatic plumbing system.

Finally, the particular configuration of the various intrusions hides any record of gas release and 
prevented us from investigating their environmental impact. However, the geochemistry offers insight into magma emplacement and contamination processes, and the petrologic analyses and the numerical model of thermal diffusion explain how high-temperature contact metamorphism is reached by sedimentary rocks sandwiched between closely related sills.

Acknowledgements. This project would not have been possible without the financing and the collaboration of the Geological Survey of Northern Ireland, and especially without the support of Garth Earls during the fieldwork and discussions. We thank Francis Coeur, Bruno Lanson and Sarah Bureau at the ISTerre for their valuable technical assistance; Catherine Chauvel, Stéphane Guillot and JeanEmmanuel Martellat for their instructive comments; and Frances Lagroix, IPGP, at the Parc Saint-Maur for the magnetization analyses. Finally, we thank N. Schofield and A. Saunders who accepted to review our manuscript and gave us relevant and helpful comments.

\section{References}

AKella, J. \& WinkLER, H. G. F. 1966. Orthorhombic amphibole in some metamorphic reactions. Contributions to Mineralogy and Petrology 12, 1-12.

BARRAT, J. A. \& NeSBITT, R. W. 1996. Geochemistry of the Tertiary volcanism of Northern Ireland. Chemical Geology 129, 15-38.

Chauvel, C., Bureau, S. \& Poggi, C. 2011. Comprehensive chemical and isotopic analyses of basalt and sediment reference materials. Geostandards and Geoanalytical Research 35, 125-43.

Coffin, M. F. \& Eldholm, O. 1994. Large igneous provinces. Reviews of Geophysics 32, 1-36.

Coulon, C. 2003. Panaches, magmatisme associé et déchirure continentale. Conférence Université d'Aix Marseille.

COURTILlot, V., JAUPART, C., MANighetTi, I., TAPPONNIER, P. \& BESSE, J. 1999. On causal links between flood basalts and continental breakup. Earth and Planetary Science Letters 166, 177-95.

FARley, K. A. \& Eltgroth, S. F. 2003. An alternative age model for the Paleocene-Eocene thermal maximum using extraterrestrial ${ }^{3} \mathrm{He}$. Earth and Planetary Science Letters 208, 135-48.

FOURIER, J. 1822. Théorie Analytique de la Chaleur. Paris: Firmin Didot Père et Fils (1822). Réédition Jacques Gabay (1988).

Ganerød, M., Mckenna, C., Smethurst, M., Prestvik, T., Rousse, S., Torsvik, T., HendriKs, B. 2008. The age of the Antrim Lava Group, Northern Ireland, and its correlation to the North Atlantic Igneous Province. American Geophysical Union, Fall Meeting, abstract \#V53A-2124.

GovinDARAJU, K. 1980. Report (1980) on three GIT-IWG rock reference samples: anorthosite from Greenland, AN-G; basalte d'Essey-la-Côte, BE-N; granite de Beauvoir, MA-N. Geostandards Newsletter 4, 49-138.

HALlAM, A 1971. Mesozoic geology and the opening of the North Atlantic. Journal of Geology 79, 129-57.

HaWkes, H. E. \& Wilson, A. H. 1975. The Portrush Sill, County Antrim, Northern Ireland. Bulletin of the Geological Survey of Great Britain 51, 1-19.

HofmanN, A. W. 1988. Chemical differentiation of the Earth: the relationship between mantle, continental crust, and oceanic crust. Earth and Planetary Science Letters 90, 297-314.
Holness, M. B. \& Humphreys, M. C. S. 2003. The Traigh Bhàn na Sgùrra Sill, Isle of Mull: flow localization in a major magma conduit. Journal of Petrology 44, 196176.

HuPPERT, H. E. \& SPARKS, R. S. J. 1989. Chilled margins in igneous rocks. Earth and Planetary Science Letters 92 , 397-405.

KennetT, J. P. \& ScotT, L. D. 1991. Abrupt deepsea warming, palaeoceanographic changes and benthic extinctions at the end of the Palaeocene. Nature 353, 225-9.

LANDES, M., RitTeR, J. R. R \& READMAN, P. W. 2007. ProtoIceland plume caused thinning of Irish lithosphere. Earth and Planetary Science Letters 255, 32-40.

Lyle, P 2003. The North of Ireland. Classic Geology in Europe 5. Harpenden, UK: Terra Publishing.

McHone, J. G. \& Butler, J. R 1984. Mesozoic igneous provinces of New England and the opening of the North Atlantic Ocean. Geological Society of America Bulletin 95, 757-65.

MCKenNA, C. 2009. The age and petrogenesis of Palaeogene flood basalt volcanism in NE Ireland. 52nd Annual Irish Geological Research Meeting, February 2009.

Mitchell W. I. (ed). 2004. The Geology of Northern Ireland - Our Natural Foundation, 2nd ed. Belfast: Geological Survey of Northern Ireland (GSNI).

NORRIS, R. D. \& RöHL, U 1999. Carbon cycling and chronology of climate warming during the Palaeocene/Eocene transition. Nature 401, 775-8.

OLD, R. A 1975. The age and field relationships of the Tardree Tertiary rhyolite complex, County Antrim, N. Ireland. Bulletin of Geological Survey of Great Britain 51, 2140.

PAtTERSON, E. M 1955. The Tertiary lava succession in the Northern part of the Antrim Plateau. Proceedings of Royal Irish Academy 57B, 79-121.

PIRAJNO, F. 2004. Hotspots and mantle plumes: global intraplate tectonics, magmatism and ore deposits. Mineralogy and Petrology 82, 183-216.

PlayfaIR, J 1802. Illustrations of the Huttonian Theory of the Earth. Edinburgh: Neill, 388 pp.

RICHARDSON, W 1803. Inquiry into the consistency of Dr. Hutton's theory of the earth with the arrangement of the strata, and other phenomena on the basaltic coast of Antrim. Transactions of the Royal Irish Academy $\mathbf{9}$, 429-87.

RhÖL, U., Bralower, T. J., Norris, R. D. \& WeFER, G 2000. New chronology for the late Paleocene thermal maximum and its environmental implications. Geology 28, 927-30.

Scarrow, J. H., Curran, J. M. \& Kerr, A. C 2000. Major element records of variable plume involvement in the North Atlantic Province Tertiary flood basalts. Journal of Petrology 41, 1155-76.

SChMitz, B., PEUCKer-EhrenbrinK, B., HEILMANNClausen, C., Aberg, G., Asaro, F. \& Lee, C. A 2004. Basaltic explosive volcanism, but no comet impact, at the Paleocene-Eocene boundary: high-resolution chemical and isotopic records from Egypt, Spain and Denmark. Earth and Planetary Science Letters 225, 117.

SCHREYER, W. 1965. Synthetische und natürliche Cordierite: II. Die chemischen Zusammensetzungen nattrlicher Cordierite und ihre Abhengigkeit von den PTXBedingungen bei der Gesteinsbildung. Neues Jahrbuch für Mineralogie Abhandlungen 103, 35-79.

SCHREYeR, W. \& Yoder, H. S. 1964. The system Mgcordierite- $\mathrm{H}_{2} \mathrm{O}$ and related rocks. Neues Jahrbuch für Mineralogie Abhandlungen 101, 271-342. 
ScotT, S. D 1974. Sulfide Mineralogy, Short Course Notes. Mineralogical Society of America I, CS21-CS29.

Sigmundsson, F. 2006. Iceland Geodynamics Crustal Deformation and Divergent Plate Tectonics. New York: Springer, pp. 209.

Storey, M., Duncan, R. A. \& Swisher, C. C. 2007. Paleocene-Eocene Thermal Maximum and the opening of the northeast Atlantic. Science 316, 587-9.

Svensen, H., Planke, S., Chevallier, L., MaltheSorenssen, A., Corfu, F. \& JAMTVEIT, B 2007. Hydrothermal venting of greenhouse gases triggering Early Jurassic global warming. Earth and Planetary Science Letters 256, 554-66.

Svensen, H., Planke, S., Malthe-Sorenssen, A., JAMTVEIT, B., MYKLEBUSt, R., RASMUSSEN EIDEM, T. $\&$ REY, S. S 2004. Release of methane from a volcanic basin as a mechanism for initial Eocene global warming. Nature 429, 542-5.

TERASHIMA, S., ANDO, A., OKAI, T., KANAI, Y., TANiguchi, M., TAKIZAWA, F. \& ITOH, S. 1990. Elemental concentrations in nine new GSJ rock reference samples "sedimentary rock series". Geostandards Newsletter 14, 1-5.

Thomas, E. \& Shackelton, N. J. 1996. The PaleoceneEocene benthic foraminiferal extinction and stable isotope anomalies. In Correlation of the Early Paleogene in Northwest Europe (eds R. W. O'B. Knox, R. M. Corfield \& R. E. Dunay), pp. 401-41.Geological Society of London, Special Publication no. 101.

ThOMPson, P 1985. Dating the British Tertiary Igneous Province in Ireland by the ${ }^{40} \mathrm{Ar}-{ }^{39} \mathrm{Ar}$ stepwise degassing method. Published Ph.D. thesis, University of Liverpool.

TRIPATI, A. \& ElDERFIELD, H. 2005. Deep-sea temperature and circulation changes at the Paleocene-Eocene Thermal Maximum. Science 308, 1894-8.

White, R. S. 1989. Initiation of the Iceland plume and opening of the North Atlantic. In Extensional Tectonics and Stratigraphy of the North Atlantic Margins (eds A. J. Tankard \& H. R. Balkwill), pp. 149-54. American Association of Petroleum Geologists Memoir no. 46 .

White, R. S. \& McKenzie, D. 1995. Mantle plumes and flood basalts. Journal of Geophysical Research $\mathbf{1 0 0}$, $17543-85$

WINKLER, H. G. F 1965. Petrogenesis of Metamorphic Rocks. New York, Berlin: Springer-Verlag, 220 pp.

Zachos, J. C., Röhl, U., Schellenberg, S. A., SluiJs, A., Hodell, D. A., Kelly, D. C., Thomas, E., Nicolo, M., RAFFI, I., LOURENS, L. J., MCCARREN, H. \& KROON, D. 2005. Rapid acidification of the ocean during the Paleocene-Eocene Thermal Maximum. Science 308, 1611-15. 